Journal of Feline Medicine and Surgery

\section{Antibody response to feline panleukopenia virus vaccination in healthy adult cats}

\author{
Michèle Bergmann ${ }^{1}$, Stephanie Schwertler ${ }^{1}$, Sven Reese ${ }^{2}$, \\ Stephanie Speck ${ }^{3}$, Uwe Truyen ${ }^{3}$ and Katrin Hartmann ${ }^{1}$
}

2018, Vol 20(12) 1087-1093

(C) The Author(s) 2017

Article reuse guidelines:

sagepub.com/journals-permissions

DOI: $10.1177 / 1098612 \times 17747740$ journals.sagepub.com/home/jfms

This paper was handled and processed by the European Editorial Office (ISFM) for publication in JFMS
(S)AGE

\begin{abstract}
Objectives According to prior studies, between $25.0 \%$ and $92.8 \%$ of adult cats have antibodies against feline panleukopenia virus (FPV) and thus are likely protected against FPV infection. It is, however, unknown how healthy adult cats with different antibody titres react to FPV vaccination in the field. Therefore, the aim of the study was to measure antibody titres in healthy adult cats within a period of 28 days after vaccination against FPV and to evaluate factors that are associated with a lack of adequate response to vaccination.

Methods One hundred and twelve healthy adult cats were vaccinated with a vaccine against FPV, feline herpesvirus and feline calicivirus. Antibodies against FPV were determined before vaccination (day 0), on day 7 and day 28 after vaccination by haemagglutination inhibition $(\mathrm{HI})$. A HI titre $\geqslant 1: 40$ was defined as protective. An adequate response to vaccination was defined as a four-fold titre increase. Uni- and multivariate statistical analysis was used to determine factors associated with an adequate response.

Results Pre-vaccination antibody titres of $\geqslant 1: 40$ were present in $64.3 \%$ (72/112; 95\% confidence interval [Cl] 55.1 72.6). Only $47.3 \%$ (53/112; $95 \% \mathrm{Cl} 37.8-57.0)$ of cats had an adequate response to vaccination. Factors associated with an adequate response to vaccination were lack of previous vaccination (odds ratio [OR] 15.58; 95\% $\mathrm{Cl} 1.4-$ 179.1; $P=0.035$ ), lack of antibodies ( $\geqslant 1: 40$ ) prior to vaccination (OR 23.10;95\% Cl 5.4-98.8; $P<0.001$ ) and breed (domestic shorthair cats; OR 7.40; 95\% Cl 1.4-38.4; $P=0.017$ ).

Conclusions and relevance As none of the cats with high pre-vaccination antibody titres ( $\geqslant 1: 160)$ had an at least four-fold increase in FPV antibody titres, measurement of antibodies rather than regular revaccinations should be performed. Thus, evaluation of FPV antibody titre in cats with previous vaccinations against FPV are recommended prior to revaccination.
\end{abstract}

Accepted: 19 November 2017

\section{Introduction}

Feline panleukopenia is a frequent and commonly fatal disease in cats. ${ }^{1}$ Therefore, vaccination is strongly recommended for all cats, and feline panleukopenia virus (FPV) is considered a core vaccine component according to expert groups worldwide. ${ }^{2-6}$ Presence of antibodies in adult cats acquired through previous vaccination or exposure to field virus correlates with protection against infection. ${ }^{7}$ According to previous studies, between $25.0 \%$ and $92.8 \%$ of adult cats have antibodies and thus are likely protected against FPV..$^{8,9}$ It is so far unknown whether cats with pre-existing antibodies benefit from revaccination.

Vaccination against FPV, especially if adjuvanted, can cause local reactions, which, in turn, might result in feline injection-site sarcomas. ${ }^{10-13}$ Thus, vaccination should only be performed if a beneficial effect can be

\footnotetext{
${ }^{1}$ Clinic of Small Animal Medicine, Centre for Clinical Veterinary Medicine, LMU Munich, Germany

2Department of Veterinary Science for Anatomy, Histology and Embryology, LMU Munich, Germany

3 Institute of Animal Hygiene and Veterinary Public Health,

University of Leipzig, Leipzig, Germany
}

\section{Corresponding author:}

Michèle Bergmann Dr med vet, Clinic of Small Animal Medicine, LMU Munich, Veterinaerstrasse 13, 80539 Munich, Germany

Email: n.bergmann@medizinische-kleintierklinik.de 
expected. The aim of the study was to evaluate the response to vaccination in healthy, adult cats within a period of 28 days after FPV vaccination, and to determine factors that are associated with an adequate response to vaccination.

\section{Materials and methods}

\section{Study population}

In total, 112 cats were prospectively included in the study between April 2012 and September 2014. A minimum sample size of at least 96 cats had been estimated in a power analysis, based on an assumed antibody prevalence of $50 \%$, with a $95 \%$ confidence interval (CI) and a $10 \%$ margin of error.

All cats were presented to the Clinic of Small Animal Medicine, Centre for Clinical Veterinary Medicine, LMU Munich or a shelter in Southern Germany for vaccination. The protocol of this prospective study was approved by the Government of Upper Bavaria (reference number 55.2-1-54-2532.3-62-11).

Cats had to be clinically healthy and adult with a minimum age of 1 year. Cats were only included if their last FPV vaccination had occurred at least $>12$ months ago. Cats were excluded if they had received immunosuppressive drugs or passive immunisation during the last 4 weeks prior to vaccination. Feline immunodeficiency virus (FIV) and feline leukemia virus (FeLV) infection status was determined using a commercial ELISA (SNAP Kombi Plus FeLV/FIV antibody test; IDEXX), and positive cats were excluded from the study. Signalment of the cats is shown in Table 1.

\section{Study protocol}

Each cat received a single dose of a modified live vaccine (MLV) on day 0, containing FPV strain PLI IV with a viral titre of $10^{3.5}$ cell culture infective dose $50 \%$, as well as feline calicivirus (FCV) and feline herpesvirus (FHV-1); FCV and FHV-1 were not subject of this study.

For the detection of pre- and post-vaccination FPV antibodies, serum samples were collected on days 0,7 and 28 , and frozen at $-20^{\circ} \mathrm{C}$ until analysed. In 23 cats, no blood sample could be obtained on day 7 .

Data on signalment (age, breed, sex, neutering status, body weight), origin (breeder, private household, animal shelter, foreign country), environment (urban, rural), housing conditions (multi-/single-cat household), lifestyle (indoor, outdoor), cohabitation with dogs, stay in a cattery or participation at a cat show, vaccination status (any previous vaccinations; presence of a complete vaccination series; time since last vaccination) were collected from the owners on day 0 . Besides obtaining a detailed history, the health status of the cats was evaluated by physical examination on days 0,7 and 28 . Vaccination side effects were recorded on days 7 and 28 .
Most of the cats $(80.6 \% ; 72 / 112)$ had received a vaccination in the past. Vaccination status was unknown in four cats beyond the previous 12 months. Only $27.8 \%$ $(20 / 72)$ of the cats had received a complete vaccination series according to current guidelines. A complete vaccination series against FPV was defined as a primary FPV vaccination series with a MLV starting at an age of 6-8 weeks with subsequent booster vaccinations at 3-4 week intervals and the last vaccination by at least 16 weeks. A booster vaccination had to be given 11-13 months later. In cats older than 12 weeks, vaccination was considered complete, if they had received two vaccinations in a 3-4 week interval with a final booster after 11-13 months. After the primary vaccination series, cats had to have received subsequent revaccinations in at least 3 year intervals.

\section{Detection of antibodies by haemagglutination inhibition}

Antibodies of all cats were measured at the end of the study. Serum samples were heat inactivated at $56^{\circ} \mathrm{C}$ for 30 mins and diluted 1:5 in barbital-acetate buffer $(\mathrm{pH}$ 6.2 ; barbituric acid $0.16 \mathrm{M}$, sodium acetate $0.143 \mathrm{M}$, $\left.\mathrm{NaCl} 1.46 \mathrm{M}, \mathrm{MgCl}_{2} \times 6 \mathrm{H}_{2} \mathrm{O} 1 \mathrm{M}, \mathrm{CaCl}_{2} \times 2 \mathrm{H}_{2} \mathrm{O} 0.3 \mathrm{M}\right)$. In preparation for the haemagglutination inhibition (HI), $500 \mu \mathrm{l}$ of diluted sera was mixed with $15 \mu \mathrm{l}$ of a $50 \%$ porcine erythrocyte suspension and were incubated for $1 \mathrm{~h}$ at $4^{\circ} \mathrm{C}$. Afterwards, sera were retrieved by centrifugation and the erythrocyte pellets were discarded. Then, sera were two-fold serially diluted in barbital-acetate buffer and mixed with an equal volume of FPV, strain 292 (eight haemagglutinating units $/ \mathrm{ml}$ ). After an incubation period of $1.5 \mathrm{~h}$ at $37^{\circ} \mathrm{C}, 50 \mu \mathrm{l} 0.5 \%$ porcine erythrocyte suspension was added. Samples were subsequently incubated overnight at $4^{\circ} \mathrm{C}$ and evaluated visually. A positive in-house control (v412/07, vaccinated cat, titre 1:640) and negative in-house control serum (FPV antibody-negative cat, titre $<1: 10$ ) were included. The endpoint was characterised as the highest serum dilution that completely inhibited haemagglutination of FPV antigen. Antibody titres of $\geqslant 1: 40$ were considered as protective against FPV.7,14,15 An at least four-fold titre increase (two titre steps) was defined as an adequate response to vaccination. ${ }^{15}$

\section{Statistical analysis}

Statistical analysis was performed with SPSS version 22 (IBM). For determination of CIs, an exact binomial test was used. ${ }^{16}$ The exact binomial test was one-tailed and used to prove the alternative hypothesis that the ratio of an adequate response to vaccination was within the $95 \%$ CI.

The $\chi^{2}$ test was used to assess risk factors associated with lack of an adequate response to vaccination. Evaluated risk factors are listed in Table 1. In case of an 
Table 1 Characteristics of cats and association with an at least four-fold titre increase during the course of the study

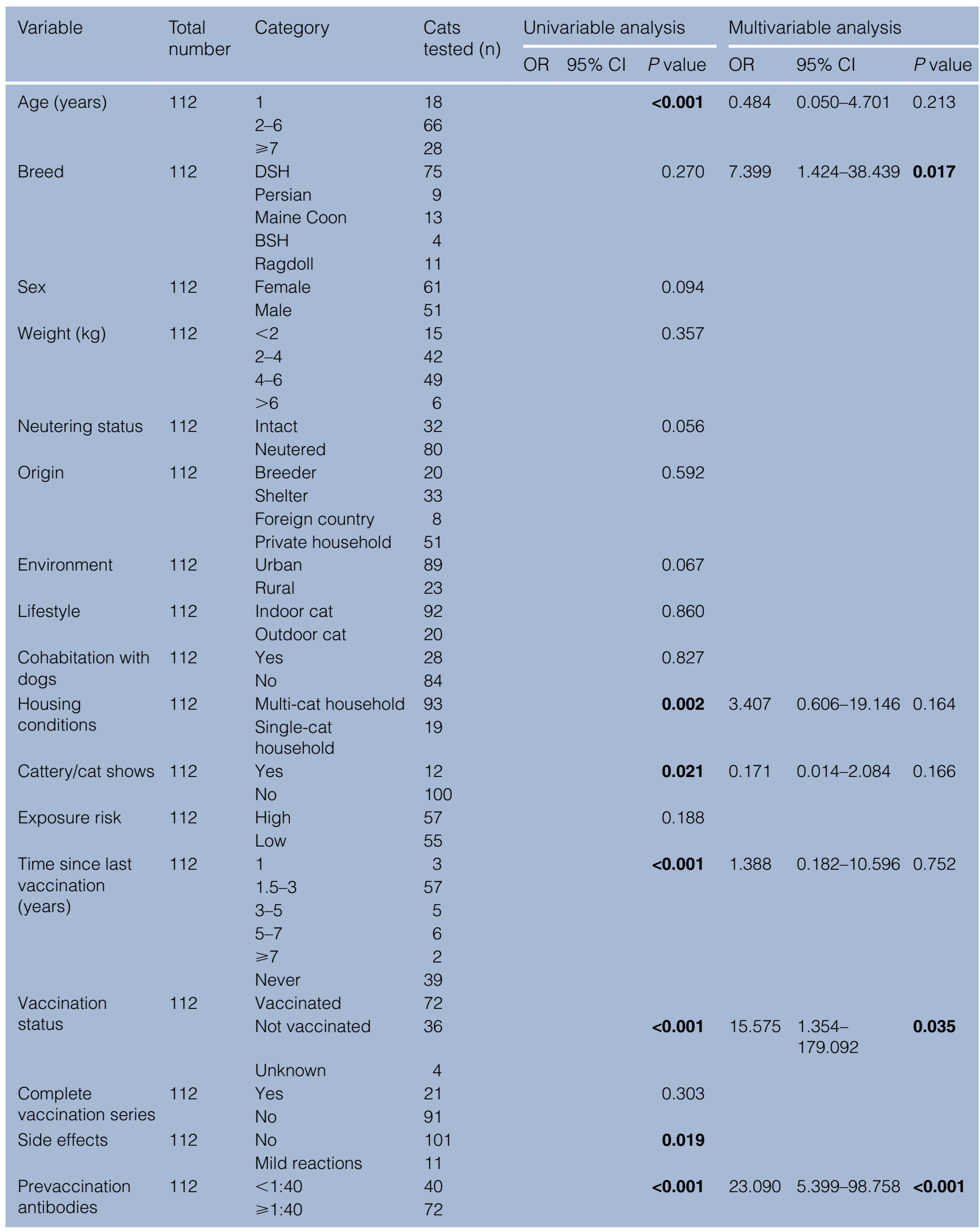

$\mathrm{DSH}=$ domestic shorthair; $\mathrm{BSH}=$ British Shorthair; $\mathrm{OR}=$ odds ratio; $\mathrm{Cl}$ = confidence interval Values in bold indicate $P<0.05$ 
expected frequency of less than five in one of the cells in the contingency table, Fisher's exact test was used. Multivariate logistic regression analysis was performed for significant factors at $P=0.05$ in univariate analysis with backwards stepwise selection based on Wald.

\section{Results}

Response to vaccination

Antibody titres of $\geqslant 1: 40$ on day 0 were present in $64.3 \%$ (72/112; 95\% CI 55.1-72.6) of the cats. An adequate response to vaccination ( $\geqslant$ two titre steps) was observed in $48.3 \%$ (54/112; $95 \%$ CI 37.8-57.0) of the cats (Table 2). Almost half of the cats showed an adequate response to vaccination by 7 days after vaccination $(40.7 \% ; 22 / 54)$.

According to their antibody response to vaccination, cats were categorised into five different groups (Figure $1)$. Cats in group $1(n=33)$ had antibodies $<1: 40$ on day 0 , and showed an at least four-fold increase $(29.5 \%$; median titre day 0: 1:10 [range 0-1:20]; median titre day 7: 1:320 [range 1:10-1:1280]; median titre day 28: 1:1280 [range 1:320-1:10240]). In group $2(\mathrm{n}=31)$, cats already had antibodies $\geqslant 1: 40$ on day 0 and showed an increase of their antibody titres after vaccination $(27.7 \%$; median titre day 0: 1:160 [range 1:40-1:1280]; median titre day 7: 1:640 [range 1:40-1:10240]; median titre day 28: 1:1280 [range 1:80-1:10240]). Group 3 consisted of five cats with antibody titres remaining $<1: 40$ pre- and postvaccination (4.5\%; median titre day 0 , day 7 and day 28 : 1:20 [range $0-1: 20$ ]). Group 4 consisted of 28 cats that had a pre-vaccination antibody titre $\geqslant 1: 40$ on day 0 and showed no titre increase after vaccination $(24.1 \%$; median titre day 0 , day 7 and day 28: 1:320 [range 1:80-1:10240]). In group $5(\mathrm{n}=15)$, cats showed an increase in their antibody titre on day 7 but a decrease on day 28 (median titre day 0: 1:1280 [range 0-1:2560]; median titre day 7: 1:2560 [range 1:320-1:5120]; median titre day 28: 1:640 [range 1:320-1:2560]).

\section{Factors associated with an adequate response to vaccination}

In univariate analysis, the factors age, housing conditions, cat show / cattery, time since last vaccination, presence of any vaccination, adverse effects and pre-vaccination antibodies were significantly associated with an adequate response to vaccination. However, in multivariate analysis only presence of any vaccination, pre-vaccination antibodies and breed proved to be significant (Table 1).

Cats without any previous vaccinations were more likely to achieve an adequate response to vaccination (odds ratio $[\mathrm{OR}] 15.58 ; P=0.035$ ) than previously vaccinated cats. However, cats with a complete vaccination series did not show a significantly different response to vaccination compared with cats that were vaccinated once or several times but not according to current guidelines. Cats with a pre-vaccination titre of $<1: 40$ were more likely to respond to vaccination than cats with a higher $(\geqslant 1: 40)$ antibody titre (OR 23.09; $P<0.001)$. Domestic shorthair (DSH) cats were more likely to respond to vaccination than purebred cats (OR 7.40; $P=0.017$ ).

\section{Discussion}

In the present study, $64.3 \%$ (72/112) of cats had antibody titres of $\geqslant 1: 40$ and thus were likely protected against panleukopenia. This result is similar to data from a former study originating from the same area, ${ }^{17}$ in which $70.6 \%$ of cats had antibody titres of $\geqslant 1: 40$. Thus, more

Table 2 Feline panleukopenia virus (FPV) titre and the number of cats with at least four-fold titre increase during the course of the study

\begin{tabular}{|c|c|c|c|c|}
\hline \multirow[t]{2}{*}{ FPV titre } & \multicolumn{3}{|c|}{ Number of cats } & \multirow{2}{*}{$\begin{array}{l}\text { Number of cats with } \geqslant \text { four-fold } \\
\text { titre increase with the respective } \\
\text { basal titre on day } 0(\%)\end{array}$} \\
\hline & Day 0 & Day $7^{*}$ & Day 28 & \\
\hline 0 & 19 & 3 & 2 & $17 / 19(89.5)$ \\
\hline $1: 10$ & 3 & 2 & 0 & $3 / 3(100.0)$ \\
\hline $1: 20$ & 18 & 6 & 3 & 15/18 (83.3) \\
\hline $1: 40$ & 6 & 8 & 0 & $5 / 6(83.3)$ \\
\hline $1: 80$ & 12 & 8 & 5 & $6 / 12(50.0)$ \\
\hline $1: 160$ & 9 & 7 & 9 & $1 / 9(11.1)$ \\
\hline $1: 320$ & 11 & 17 & 17 & 2/11 (18.2) \\
\hline $1: 640$ & 10 & 8 & 17 & 2/10 (20.0) \\
\hline $1: 1280$ & 14 & 15 & 24 & $3 / 14(21.4)$ \\
\hline $1: 2560$ & 9 & 11 & 24 & $0 / 9(0.0)$ \\
\hline $1: 5120$ & 0 & 2 & 8 & 0/0 (0.0) \\
\hline $1: 10240$ & 1 & 2 & 3 & $0 / 1(0.0)$ \\
\hline No value & 0 & 23 & 0 & \\
\hline $\begin{array}{l}\text { Total number of cats with } \\
\geqslant \text { four-fold titre increase (\%) }\end{array}$ & & & & $54(48.3)$ \\
\hline
\end{tabular}




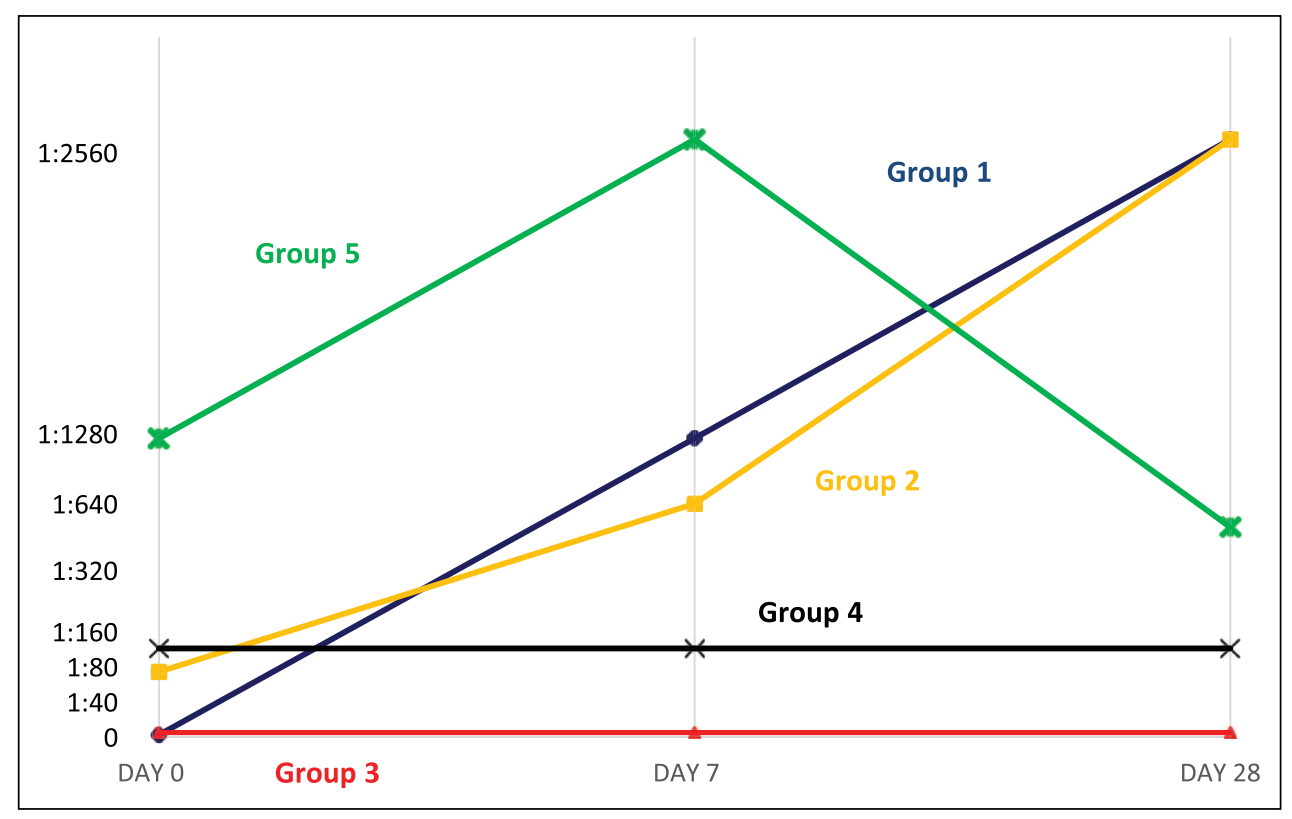

Figure 1 Categorisation of cats into five groups depending on median feline panleukopenia virus (FPV) antibody titres and on antibody response to vaccination against FPV. Vertical axis shows cats' median antibody titre against FPV; horizontal axis shows median antibody titres throughout the study, day 0 (before vaccination), day 7 and day 28 (after vaccination). FPV titre could not be determined in 23 cats on day 7 . Group $1=$ cats without antibodies $<1: 40$ on day 0 and an adequate antibody titre increase on day 7 and/or day $28(n=33 ; 29.5 \%)$; group $2=$ cats with antibodies $\geqslant 1: 40$ and any titre increase $(n=31 ; 27.7 \%)$; group $3=$ cats with antibody titre remaining $<1: 40$ pre- and post-vaccination $(n=5 ; 4.5 \%)$; group $4=$ cats with pre-vaccination antibody titre $\geqslant 1: 40$ on day 0 and no titre increase after vaccination $(n=28 ; 25.0 \%)$; group $5=$ cats with antibody titre increase on day 7 but decrease on day $28(n=15 ; 13.4 \%)$

than one-third of the cats had no antibodies or low titres. In contrast, the prevalence of antibody titres against canine parvovirus (CPV) in dogs in the same area is much higher $(86.0 \%) .{ }^{18}$ This is probably because of natural booster effects through more intensive contact of dogs to $\mathrm{CPV}$, resulting in common inapparent infections in dogs. This was also demonstrated in one study, in which 2/100 healthy dogs shed virus in their faeces but showed no signs of disease. ${ }^{19}$ In contrast to dogs, cats have a solitary lifestyle and bury their faeces, or they are kept indoors only and have no contact to dog faeces. Natural boosters, therefore, are less likely in cats.

An adequate response to vaccination was observed in $48.3 \%$ of the cats. The remaining cats did not respond adequately. In a comparable study in dogs, ${ }^{18}$ only $17.0 \%$ of the dogs reacted adequately to vaccination. This difference can mainly be explained by the difference in pre-existing antibodies. Most of the dogs had high pre-vaccination antibodies and did not react adequately to vaccination. Riedl et al found that an inadequate response to vaccination in dogs was associated with higher body weight; dogs $>10 \mathrm{~kg}$ were more likely not to react to vaccination. ${ }^{18}$ Cats in the present study weighed between 1.7 and $7.1 \mathrm{~kg}$ (mean $3.9 \mathrm{~kg}$ ) and in contrast to dogs, weight had no influence on response to vaccination.

Response to vaccination followed five different reaction schemes. Interestingly, five cats with no or low pre-existing antibodies did not respond to vaccination. These cats were regarded as non-responders. Nonresponders are well known in veterinary medicine, $, 15,18,20-22$ as well as in human medicine, especially after vaccination against hepatitis B virus.

Several factors can be responsible for an absence of response to vaccination. ${ }^{23,24}$ In humans, smoking and chronic diseases, like diabetes and chronic kidney disease, are described to be responsible for a lack of antibody production. ${ }^{25,26}$ However, cats of the present study had an unremarkable history and were healthy in physical examination and FIV/FeLV negative. Other reasons for an inadequate response to vaccination that cannot be excluded in the cats of the present study include genetic variations or an immune system that does not recognise the vaccine antigen..$^{23}$ Wrong administration or impaired vaccine storage, leading to an inactivation of the MLV, could also play a role. ${ }^{23,24}$ However, this is very unlikely in the present study as vaccination was always performed by the same person. Obesity can be a cause of inadequate response to vaccination. ${ }^{23,24}$ However, none of the cats included in the present study were obese. Furthermore, cats might not have displayed a humoral response but developed a cellular response, which was not evaluated.

Cats in group $5(\mathrm{n}=16)$ showed another interesting phenomenon. They had pre-existing antibody titres on day 0 and a titre increase on day 7 , but the titre decreased 
again on day 28. The reason for this antibody decrease is unknown. A possible explanation might be the binding of the pre-existing antibodies to the vaccine virus. It would be interesting to measure antibody levels after day 28 to see if the titre would stay at that level, decrease further or increase again. As the study was only designed until day 28, those samples were not obtained.

An adequate response to vaccination in the present study was associated with: (1) having never been vaccinated at all; (2) having a low pre-vaccination titre; and (3) being DSH. In dogs, it is known that some breeds (eg, Rottweilers) are more likely to react less effectively to vaccination (eg, CPV or rabies). ${ }^{18,21,22,23,27}$ As FPV outbreaks have been reported in Norwegian Forest Cats (NFCs) in the past, the possibility of NFCs failing to react adequately to vaccination has been discussed. However, a study comparing NFC kittens with DSH kittens showed no difference in their response to FPV vaccination. ${ }^{28}$ To date, it is still unknown whether different cat breeds might be predisposed for vaccination failure. Owing to the small cat number in specific breeds in the present study, no conclusion on specific breeds was possible and further studies are necessary to evaluate breed predisposition towards vaccination failure in cats.

The pre-vaccination antibody titre of cats was significantly associated with an adequate response to vaccination. Cats with high antibodies probably neutralise the vaccine virus before it stimulates the immune system. The mechanism is known from kittens with maternally derived antibodies that commonly interfere with active immunisation. ${ }^{28,29}$ A study in dogs showed similar results. ${ }^{18}$ Low antibody titres in cats, as well as in dogs, were more likely associated with an adequate response to vaccination. Interestingly, a complete vaccination series was not decisive for an adequate response to vaccination. In contrast, the crucial fact was whether the cat had ever been vaccinated or not.

This raises the question whether a complete vaccination series is really necessary if using MLV. In dogs, a single vaccination against $\mathrm{CPV}$ is likely sufficient to induce immunity. ${ }^{4}$ Depending on the response to vaccination in cats of the present study, one dose might be enough for production of sufficient antibodies by the immune system. To minimise severe vaccination adverse effects, such as injection site-associated sarcomas, regular testing of antibodies instead of vaccination could be advised. The antibody titre that should be used as cut-off and below which re-vaccination should be performed still needs to be determined. So far, re-vaccination against parvovirosis is recommended for cats with titres $<1: 40 .{ }^{30}$ However, adult cats are likely to be protected even if circulating antibodies fall below these levels. Commercial in-house tests are useful when used as part of regular health check appointments in veterinary practice or for the control of a successful primary vaccination series against parvovirosis. ${ }^{31}$
In the present study, only $14.8 \%(8 / 54)$ cats with an antibody titre of $\geqslant 1: 160$ on day 0 responded to vaccination vs $79.3 \%(46 / 58)$ with a titre $\leqslant 1: 80$. Overall, $83.3 \%$ $(30 / 36)$ of the cats without previous vaccinations, showed an adequate response to FPV vaccination. Interestingly, $16 / 36$ of the cats without previous vaccinations had antibody titres $\geqslant 1: 40$ on day 0 (range 1:40-1:1280). This indicates that natural exposure is an important source of immunisation also in cats. Antibody titres are probably derived from previous contacts to FPV or CPV from the cats' surroundings. CPV is known to infect cats and also to cause disease. ${ }^{32-34}$ Parvoviruses can survive up to 1 year in the environment and are therefore able to infect even indoor-only cats when being transmitted through fomites. ${ }^{35}$

There are a few limitations to the study. FPV titre could not be determined in all cats on day 7 . Validity of the cats' history depended on owners' reports and thus might not always have been correct. In addition, it is not proven that lack of antibody titre increase is equivalent to a lack of development of protection against disease, as antibodies are not the only source of protection. Cell-mediated immunity can also be an effective protection. However, challenge studies would be needed to prove this hypothesis, which cannot be undertaken in privately owned cats.

\section{Conclusions}

Fifty-four of 112 (48.2\%) cats reacted appropriately to vaccination. Although this rate is much higher than in dogs, it still means that half of the cats do not benefit from vaccination. Factors associated with an adequate response to vaccination were lack of a non-protective pre-vaccination titre, having never been vaccinated before and DSH breed. Therefore, evaluation of FPV antibody titre in cats with previous vaccination history against FPV is recommended prior to regular revaccinations. Especially in cats with a high titre (such as $\geqslant 1: 160$ ), re-vaccinations against FPV are not beneficial and therefore unnecessary. If vaccination against other infectious agents is considered necessary, veterinarians should consider using vaccines that do not contain a FPV component, which are also available on the market.

Acknowledgements We would like to thank Mrs Nadja Leinecker from the Institute of Animal Hygiene and Veterinary Public Health, University of Leipzig, for her advice, expertise and processing of the samples. We thank Merial for funding and supporting this study, and especially Jean-Christophe Thibault for his valuable input.

Author note Parts of the results were presented as an abstract ( $<250$ words) and oral presentation at the 25th ECVIM-CA Congress in Lisbon, Portugal, 2015. Some of the results were also presented as an abstract ( $<250$ words) and poster at the 23rd annual conference of the German Society of Internal Medicine and Clinical Pathology of the German Veterinary Association (DVG) in Leipzig, Germany, 23-24 January 2015. 
Conflict of interest This study was funded by Merial, Lyon, France. Merial played no role in the collection and interpretation of data, or in the decision to submit the manuscript for publication. There is no commercial conflict of interest as the information generated here is solely for scientific dissemination.

Funding This study was funded by Merial, Lyon, France.

\section{References}

1 Kruse BD, Unterer S, Horlacher K, et al. Prognostic factors in cats with feline panleukopenia. J Vet Intern Med 2010; 24: 1271-1276.

2 Richards JR, Elston TH, Ford RB, et al. The 2006 American Association of Feline Practitioners Feline Vaccine Advisory Panel report. J Am Vet Med Assoc 2006; 229: 1405-1441.

3 Truyen U, Addie D, Belak S, et al. Feline panleukopenia. ABCD guidelines on prevention and management. J Feline Med Surg 2009; 11: 538-546.

4 Day MJ, Horzinek MC, Schultz RD, et al. WSAVA guidelines for the vaccination of dogs and cats. J Small Anim Pract 2016; 57: 1-45.

5 Ständige Impfkommission Vet. Leitlinie zur Impfung von Kleintieren. Deutsches Tierärzteblatt 2013; 7.

6 Hosie MJ, Addie DD, Boucraut-Baralon C, et al. Matrix vaccination guidelines: $2015 \mathrm{ABCD}$ recommendations for indoor/outdoor cats, rescue shelter cats and breeding catteries. J Feline Med Surg 2015; 17: 583-587.

7 Lappin MR, Andrews J, Simpson D, et al. Use of serologic tests to predict resistance to feline herpesvirus 1 , feline calicivirus, and feline parvovirus infection in cats. $J \mathrm{Am}$ Vet Med Assoc 2002; 220: 38-42.

8 Blanco K, Prendas J, Cortes R, et al. Seroprevalence of viral infections in domestic cats in Costa Rica. J Vet Med Sci 2009; 71: 661-663.

9 Hellard E, Fouchet D, Santin-Janin H, et al. When cats' ways of life interact with their viruses: a study in 15 natural populations of owned and unowned cats (Felis silvestris catus). Prev Vet Med 2011; 101: 250-264.

10 Hendrick MJ and Goldschmidt MH. Do injection site reactions induce fibrosarcomas in cats? J Am Vet Med Assoc 1991; 199: 968.

11 Lester S, Clemett T and Burt A. Vaccine site-associated sarcomas in cats: clinical experience and a laboratory review (1982-1993). J Am Anim Hosp Assoc 1996; 32: 91-95.

12 Burton G and Mason KV. Do postvaccinal sarcomas occur in Australian cats? Aust Vet J 1997; 75: 102-106.

13 Hartmann K, Day MJ, Thiry E, et al. Feline injection-site sarcoma: ABCD guidelines on prevention and management. J Feline Med Surg 2015; 17: 606-613.

14 Scott FW and Geissinger CM. Long-term immunity in cats vaccinated with an inactivated trivalent vaccine. $A m \mathrm{~J}$ Vet Res 1999; 60: 652-658.

15 Mouzin DE, Lorenzen MJ, Haworth JD, et al. Duration of serologic response to three viral antigens in cats. $J \mathrm{Am}$ Vet Med Assoc 2004; 224: 61-66.

16 Clopper CJ and Pearson ES. The use of confidence or fiducial limits illustrated in the case of the binomial. Biometrika 1934; 26: 404-413.
17 Mende K, Stuetzer B, Sauter-Louis C, et al. Prevalence of antibodies against feline panleukopenia virus in clientowned cats in Southern Germany. Vet J 2014; 199: 419-423.

18 Riedl M, Truyen U, Reese S, et al. Prevalence of antibodies to canine parvovirus and reaction to vaccination in clientowned, healthy dogs. Vet Rec 2015; 177: 597.

19 Riedl M, Truyen U, Speck S, et al. Faecal shedding of canine parvovirus following modified live vaccination in adult, healthy dogs. Vet $J$ 2017; 219: 15-21.

20 Larson LJ and Schultz RD. Three-year duration of immunity in dogs vaccinated with a canarypox-vectored recombinant canine distemper virus vaccine. Vet Ther 2007; 8: 101-106.

21 Glickman LT, Domanski LM, Patronek GJ, et al. Breedrelated risk factors for canine parvovirus enteritis. $J \mathrm{Am}$ Vet Med Assoc 1985; 187: 589-594.

22 Houston DM, Ribble CS and Head LL. Risk factors associated with parvovirus enteritis in dogs: 283 cases (1982-1991). J Am Vet Med Assoc 1996; 208: 542-546.

23 Horzinek MC and Thiry E. Vaccines and vaccination: the principles and the polemics. J Feline Med Surg 2009; 11: 530-537.

24 Greene CE and Levy JK. Immunoprophylaxis. In: Greene CE (ed). Infectious diseases of the dog and cat. 4th ed. St Louis, MO: Elsevier-Saunders, 2012, pp 1163-1205.

$25 \mathrm{Yu}$ AS, Cheung RC and Keeffe EB. Hepatitis B vaccines. Infect Dis Clin North Am 2006; 20: 27-45.

26 Filippelli M, Lionetti E, Gennaro A, et al. Hepatitis B vaccine by intradermal route in non responder patients: an update. World J Gastroenterol 2014; 20: 10383-10394.

27 Kennedy LJ, Lunt M, Barnes A, et al. Factors influencing the antibody response of dogs vaccinated against rabies. Vaccine 2007; 12: 8500-8507.

28 Jakel V, Cussler K, Hanschmann KM, et al. Vaccination against feline panleukopenia: implications from a field study in kittens. BMC Vet Res 2012; 8: 62.

29 Scott FW, Csiza CK and Gillespie JH. Maternally derived immunity to feline panleukopenia. J Am Vet Med Assoc 1970; 156: 439-453.

30 Digangi BA, Gray LK, Levy LK, et al. Detection of protective antibody titers against feline panleukopenia virus, feline herpesvirus-1, and feline calicivirus in shelter cats using a point-of-care ELISA. J Feline Med Surg 2011; 13: 912-918.

31 Mende K, Stuetzer B, Truyen U, et al. Evaluation of an in-house dot enzyme-linked immunosorbent assay to detect antibodies against feline panleukopenia virus. J Feline Med Surg 2014; 16: 805-811.

32 Truyen U, Gruenberg A, Chang SF, et al. Evolution of the feline-subgroup parvoviruses and the control of canine host range in vivo. J Virol 1995; 69: 4702-4710.

33 Truyen U. Evolution of canine parvovirus: loss and gain of the feline host [article in German]. Tierarztl Prax 1996; 24: 316-318.

34 Mochizuki M, Horiuchi $\mathrm{M}$, Hiragi $\mathrm{H}$, et al. Isolation of canine parvovirus from a cat manifesting clinical signs of feline panleukopenia. J Clin Microbiol 1996; 34: 2101-2105.

35 Uttenthal A, Lund E and Hansen M. Mink enteritis parvovirus. Stability of virus kept under outdoor conditions. APMIS 1999; 107: 353-358. 